\title{
Hypertension and type 2 diabetes: lights and shadows about causality
}

\author{
GianLuca Colussi $i^{1} \cdot$ Andrea Da Porto $^{1} \cdot$ Alessandro Cavarape $^{1}$
}

Received: 24 July 2019 / Revised: 2 September 2019 / Accepted: 24 September 2019 / Published online: 21 October 2019

(C) Springer Nature Limited 2019

Hypertension and type 2 diabetes (T2D) are closely associated with each other, but whether a direct causal effect links the two conditions is unclear. A recent Mendelian randomization (MR) analysis on over 300,000 subjects free of cardiovascular disease has provided some evidence. While T2D is causal for hypertension development, the same is not proven for the reversed relationship. Further studies should disentangle the complex network of molecular pathways that link hypertension and T2D. Specific targets with causal relevance to preventing the codevelopment of the two conditions are needed, and hyperinsulinemia might be a good candidate.

According to the World Health Organization, after obesity, arterial hypertension and T2D are the two cardiovascular risk factors most prevalent in the global population. This is remarkable because both diseases are responsible for the high number of cardiovascular events and deaths worldwide [1]. In the last 30 years, the prevalence of hypertension has decreased to one-fourth of the global population [2], but that of diabetes has continued to increase from 4.7 to $8.5 \%$, and a projection for the future shows another dramatic increment [3]. Improving awareness, treatment, and control of these diseases is a major goal of the global health system.

Despite their single roles as independent cardiovascular risk factors, hypertension and T2D often coexist in the same patient. This coexistence multiplies the patient's risk of experiencing major acute cardiovascular events and accelerates the development of chronic heart and kidney failure [4]. Authors have analyzed the simultaneous presence of hypertension and T2D in Western countries and in Japan. It emerged that $20 \%$ of patients with hypertension had T2D and $50 \%$ of patients with T2D had hypertension. Having

GianLuca Colussi

gianluca.colussi@uniud.it

1 Division of Internal Medicine, Department of Medicine, University of Udine, 33100 Udine, Italy one of the two conditions increases the risk of developing the other by 1.5-2.0 times [5]. However, which condition develops first, increasing the risk of developing the other, remains unclear.

Prospective studies have documented a potential reciprocal relationship between hypertension and T2D in which they elicit each other. Nondiabetic hypertensive patients have a high prevalence of prediabetes [6], and the incidence of T2D in the general population is high as baseline blood pressure values are generally elevated [7]. A meta-analysis of 30 prospective studies confirmed that each $20 \mathrm{~mm} \mathrm{Hg}$ increment of systolic blood pressure increased the risk of new-onset T2D by 77\% [7]. Other studies analyzed risk factors for developing T2D in patients with hypertension. In these studies, fasting plasma glucose, body mass index, and the class of antihypertensive drugs used were independent determinants of T2D [8]. T2D can also be a risk factor for developing hypertension. In the Multi-Ethnic Study of Atherosclerosis, baseline T2D, high fasting glucose levels, and high insulin levels were independent risk factors for hypertension [9]. Although hypertension and T2D conditions influence each other reciprocally, the nature of this relationship remains to be defined.

Hypertension and T2D share several pathophysiological mechanisms that justify their coexistence. Insulin resistance, hyperinsulinemia, increased oxidative stress, and subclinical chronic inflammation are the most studied mechanisms. Hyperinsulinemia is induced by insulin resistance and predicts hypertension development in normotensive nondiabetic patients [10]. Hyperinsulinemia causes arterial remodeling and arterial stiffness, increases sympathetic nervous activity, enhances the renin-angiotensin-aldosterone system, and stimulates renal sodium reabsorption [11]. In addition, excessive oxidative stress and inflammation are determinants of endothelial dysfunction, impaired vascular reactivity, increased peripheral vascular resistance, and abnormal glucose and lipid metabolism. All mechanisms that lead to increased levels of blood pressure and plasma glucose by changing hemodynamic and glycolipid metabolism [12]. 
However, these mechanisms have been documented in only experimental and observational studies, limiting the relevance of the causal effects. Unmeasured confounders, selection bias, and reverse causation make the causal interaction between hypertension and T2D uncertain. Recently, however, a mendelian randomization (MR) analysis by Sun et al. contributed to providing an answer [13].

MR analysis comprises instrumental variables derived from the genetic characterization of individuals by genome-wide association studies (GWAS). The variable is built by the variants of genes (single nucleotide polymorphisms-SNPs) that are genetic determinants of the exposure factor. Because of the random distribution of genes at individual conception, sorting individuals by genetic variables should eliminate confounders. In fact, similar to a randomized controlled trial, in an MR analysis, selection bias and risk of reverse causation are attenuated. Pleiotropic bias is the risk of an instrumental variable being associated with the outcome of interest via other indirect pathways or with traits in linkage disequilibrium with the exposure of interest. Several statistical methods have been developed to account for pleiotropy in MR analysis and were used in Sun's work [14].

Sun et al. performed a bidirectional MR analysis using hundreds of SNPs correlated to hypertension as an instrumental variable to predict $\mathrm{T} 2 \mathrm{D}$ and vice versa. They used GWAS data from the UK Biobank, which comprises genotypes of over 300,000 adults. The included subjects were of both sexes, of European descent, and free of cardiovascular diseases. They found that genetically predisposed T2D was related to a $7 \%$ increased risk of hypertension. T2D was associated with a $0.67 \mathrm{mmHg}$ increase in systolic blood pressure, but it did not affect diastolic blood pressure. Conversely, genetically predisposed hypertension or elevated blood pressure levels were not related to T2D. The authors detected significant pleiotropy in the relationship between hypertension and the development of T2D [13].

This analysis shows that diabetes is a causal factor for developing hypertension but not vice versa. Therefore, reducing hyperglycemia to prevent hypertension could be a target, while reducing blood pressure to prevent T2D could not. Nevertheless, the causal effect of T2D on the development of hypertension is miniscule and, as such, not clinically relevant. In both cases, developing T2D or hypertension, as predicted by the genetic variants, does not justify the strong association observed between the two conditions per se. Different causal factors should be considered in the development of T2D in patients with hypertension and vice versa. Hyperinsulinemia, abdominal adiposity, inflammation, and the class of antihypertensive drugs can be of more relevance than hypertension itself in the development of T2D. On the other hand, hyperinsulinemia, arterial stiffness, renal dysfunction, and hyperglycemia can contribute to hypertension $[8,15]$.

In conclusion, the presence of T2D is a causal factor in the development of hypertension. Although the effect is miniscule, the result is robust and explains the development of high blood pressure in patients with T2D. The evidence is null when the relationship is reversed but, in both cases, we are far from a full causal explanation. We should search for more relevant factors in the complex network of interconnected conditions associated with hypertension and T2D. This complex network needs to be disentangled to identify specific pathways, and MR analysis can provide valid support for the identification of such pathways. The aim of future studies should identify specific targets with causal relevance to prevent the codevelopment of hypertension and T2D. Hyperinsulinemia might be a good candidate.

\section{Compliance with ethical standards}

Conflict of interest The authors declare that they have no conflict of interest.

Publisher's note Springer Nature remains neutral with regard to jurisdictional claims in published maps and institutional affiliations.

\section{References}

1. Roth GA, Huffman MD, Moran AE, Feigin V, Mensah GA, Naghavi M, et al. Global and regional patterns in cardiovascular mortality from 1990 to 2013. Circulation. 2015;132:1667-78.

2. Zhou B, Bentham J, Di Cesare M, Bixby H, Danaei G, Cowan MJ, et al. Worldwide trends in blood pressure from 1975 to 2015: a pooled analysis of 1479 population-based measurement studies with 19.1 million participants. Lancet. 2017;389:37-55.

3. Lin J, Thompson TJ, Cheng YJ, Zhuo X, Zhang P, Gregg E, et al. Projection of the future diabetes burden in the United States through 2060. Popul Health Metr. 2018;16:9.

4. Verdecchia P, Reboldi G, Angeli F, Borgioni C, Gattobigio R, Filippucci L, et al. Adverse prognostic significance of new diabetes in treated hypertensive subjects. Hypertension. 2004; 43:963-9.

5. Tatsumi Y, Ohkubo T. Hypertension with diabetes mellitus: significance from an epidemiological perspective for Japanese. Hypertens Res. 2017;40:795-806.

6. Catena C, Colussi G, Martinis F, Pezzutto F, Sechi LA. Plasma glucose levels and left ventricular diastolic function in nondiabetic hypertensive patients. Am J Hypertens. 2013;26:1353-61.

7. Emdin CA, Anderson SG, Woodward M, Rahimi K. Usual blood pressure and risk of new-onset diabetes: evidence from 4.1 million adults and a meta-analysis of prospective studies. J Am Coll Cardiol. 2015;66:1552-62.

8. Aksnes TA, Kjeldsen SE, Rostrup M, Störset Ö, Hua TA, Julius S. Predictors of new-onset diabetes mellitus in hypertensive patients: the VALUE trial. J Hum Hypertens. 2008;22:520-7.

9. Levin G, Kestenbaum B, Ida Chen Y-D, Jacobs DR, Psaty BM, Rotter JI, et al. Glucose, insulin, and incident hypertension in the multi-ethnic study of atherosclerosis. Am J Epidemiol. 2010; 172:1144-54. 
10. Park SE, Rhee E-J, Park C-Y, Oh KW, Park S-W, Kim S-W, et al. Impact of hyperinsulinemia on the development of hypertension in normotensive, nondiabetic adults: a 4-year follow-up study. Metabolism. 2013;62:532-8.

11. Ferrannini E, Cushman WC. Diabetes and hypertension: the bad companions. Lancet. 2012;380:601-10.

12. Petrie JR, Guzik TJ, Touyz RM. Diabetes, hypertension, and cardiovascular disease: clinical insights and vascular mechanisms. Can J Cardiol. 2018;34:575-84.
13. Sun D, Zhou T, Heianza Y, Li X, Fan M, Fonseca VA, et al. Type 2 diabetes and hypertension. Circ Res. 2019;124:930-7.

14. Hemani G, Bowden J, Davey Smith G. Evaluating the potential role of pleiotropy in Mendelian randomization studies. Hum Mol Genet. 2018;27:R195-R208.

15. Tsimihodimos V, Gonzalez-Villalpando C, Meigs JB, Ferrannini E. Hypertension and diabetes mellitus: coprediction and time trajectories. Hypertension. 2018;71:422-8. 